\title{
Cost-effectiveness analysis of mechanical thrombectomy in acute ischemic stroke
}

\author{
CHIRAG G. PATIL, M.D. ${ }^{1}$, ELISA F. LONG, M.S. ${ }^{2}$, and MAARTEN G. LANSBERG, M.D., PH.D. \\ 3 \\ 1 Department of Neurosurgery, Stanford University School of Medicine, Stanford University, Stanford \\ 2Department of Management Science \& Engineering, Stanford University, Stanford \\ 3Stanford Stroke Center, Stanford University Medical Center, Palo Alto, California
}

\begin{abstract}
Object-Mechanical thrombectomy is increasingly being used for the treatment of large-vessel ischemic stroke in patients who arrive outside of the 3-hour tissue plasminogen activator time window. In this study, the authors evaluated the cost and effectiveness of mechanical thrombectomy compared with standard medical therapy in patients who are ineligible to receive tissue plasminogen activator.
\end{abstract}

Methods-Clinical outcomes of an open-label study of mechanical thrombectomy were compared with a hypothetical control group with a lower recanalization rate (18 vs 60\%) and a lower rate of symptomatic intracranial hemorrhage ( 0.6 vs $7.8 \%$ ) than the active treatment group. A Markov costeffectiveness model was built to compare the health benefits and costs associated with mechanical thrombectomy compared with standard medical therapy. All probabilities, quality-of-life factors, and costs were estimated from the published literature. Univariate sensitivity analyses were performed to assess how variations in model parameters affect health and economic outcomes.

Results-Treatment of acute ischemic stroke with mechanical thrombectomy increased survival time by 0.54 quality-adjusted life years (QALYs), compared with standard medical therapy ( $2.37 \mathrm{vs}$ 1.83 QALYs), at an increased cost of $\$ 6600$. This yielded an incremental cost-effectiveness ratio (ICER) of $\$ 12,120$ per QALY gained, a value generally considered cost-effective. Sensitivity analysis showed that mechanical thrombectomy remained cost-effective (ICER $<\$ 50,000$ per QALY gained) for all model inputs varied over a reasonable range, except for age at stroke treatment. For patients older than 82 years of age, the treatment was only borderline cost-effective (ICER of \$50,000-100,000 per QALY gained).

Conclusions-The treatment of large-vessel ischemic stroke with mechanical thrombectomy appears to be cost-effective. These results require validation when data from a randomized, controlled trial of mechanical thrombectomy become available.

\section{Keywords}

cost-benefit analysis; cost-effectiveness; mechanical thrombectomy; stroke

EndovasculaR mechanical thrombectomy for acute ischemic stroke is being increasingly performed by neurosurgeons and neurointerventionalists. Mechanical thrombectomy devices reopen large cerebral vessels and represent an alternative treatment to the standard medical

Address correspondence to: Chirag G. Patil, M.D., Stanford Hospital, Department of Neurosurgery, 300 Pasteur Drive, Room R200 MC5327, Stanford, California 94305. email: E-mail: chiragpatil@gmail.com.. 
therapy with antiplatelet agents in patients with large-vessel ischemic stroke who are not eligible for thrombolytic therapy, or in whom thrombolytic therapy has failed. The Merci retriever system (Concentric Medical) and more recently, the Penumbra system (Penumbra Inc.) have been granted $510(\mathrm{k})$ clearance by the US FDA for use in revascularization in patients with acute stroke within 8 hours of symptom onset. The FDA's approval of these devices was based on studies in which higher recanalization rates after mechanical thrombectomy (48\% for Merci; $81 \%$ for Penumbra) were demonstrated compared with the rate of spontaneous recanalization (18\%) observed in the placebo arm of the PROACT II study. $7,10,13,14$ Furthermore, studies have shown superior neurological outcomes and a lower mortality rate in patients in whom recanalization is achieved compared with patients without recanalization. $7,10,13,14$

Decisions concerning the clinical utility of an intervention should be guided by information regarding the benefits, risks, and costs associated with that intervention. Cost-effectiveness analysis is a tool that can be used to assess outcomes and costs in a unified model. The objective of our analysis was to determine whether mechanical thrombectomy for acute ischemic stroke is cost-effective from a societal perspective.

\section{Methods \\ Model Overview}

To determine the cost-effectiveness of mechanical thrombectomy compared with standard medical therapy for acute ischemic stroke, we created a decision analytic model using TreeAge software (TreeAge Software, Inc). We developed a Markov state-transition model, which accounts for the possible health states an individual may enter after presenting with acute ischemic stroke (Fig. 1). We considered 2 treatment options and estimated the average healthcare costs and benefits of each from the time of stroke until death. We assumed a 20 -year time horizon to account for deaths and complications related to future recurrent strokes and agerelated causes. We used as our base case a 67 -year-old patient with large-vessel ischemic stroke.

\section{Management Strategies}

Two management strategies were considered for acute stroke in patients who could receive treatment within 8 hours of symptom onset, but were not eligible for intravenous tPA: 1) an interventional strategy consisting of mechanical thrombectomy with the Merci mechanical clot retrieval system, and 2) a reference strategy consisting of standard medical therapy with antiplatelet agents and supportive care but without the use of thrombolytics (such as tPA). The interventional strategy was chosen based on the FDA's recent approval of the Merci Retriever for removal of blood clots from cerebral arteries. The approval was based on the results of the MERCI study, ${ }^{14}$ which included only patients ineligible for intravenous tPA therapy. Standard medical management without the use of thrombolytics was therefore chosen as the reference strategy. The reference strategy is based on the American Heart Association and the American College of Chest Physicians guidelines for the management of ischemic stroke. ${ }^{2,3}$

\section{Health States}

Patients were categorized into 1 of 3 health outcome states based on their predicted mRS score 90 days after treatment: independent (mRS score $\leq 2$ ), dependent (mRS score 3-5), or dead (Fig. 1). See Model Parameters below for detailed description of model inputs. We assumed that patients remained in the same health state for the entire first year. Following the initial year and at the end of each subsequent annual cycle, patients could remain in their current health state, transition to a lower health state (due to a recurrent stroke), or die (due to a recurrent stroke or an age-related cause) At the end of each annual cycle, we updated each individual's age to account for the change in age-related mortality rate. 


\section{Outcome Assessment}

We estimated the health benefits and costs associated with treatment of acute ischemic stroke by mechanical thrombectomy and standard medical therapy. Health benefits were measured in QALYs, which captured the effects of treatment on rates of morbidity and mortality. The economic costs included the cost of hospitalization for acute stroke (such as inpatient and endovascular procedure costs), the cost of rehabilitation after stroke, and the cost of long-term care associated with independent and dependent health states. We used the Markov model to calculate the costs and QALYs associated with the initial treatment and the costs and benefits of all future health states until death. We discounted all health costs and benefits at an annual rate of $3 \% .^{8}$

The difference in costs and benefits between treatment with mechanical thrombectomy and standard stroke therapy were assessed. The ICER was calculated by dividing the difference in costs by the difference in QALYs. The intervention was considered cost-effective if the ICER was $<\$ 50,000$ per QALY gained, and borderline cost-effective if the ICER was $\$ 50,000-100,000$ per QALY gained. ${ }^{8}$ All cost-effectiveness analyses were performed from a societal perspective.

\section{Model Parameters}

Model inputs and the range over which they were tested are shown in Table 1. The base case probability of recanalization in patients who received standard medical therapy was based on the rate of recanalization observed in the placebo group of the PROACT II study. ${ }^{7}$ The base case probability of recanalization with mechanical thrombectomy was based on the MERCI trial. ${ }^{14}$ The probability of sICH with standard medical therapy was based on the control arm of the NINDS tPA stroke trial, ${ }^{1}$ and the probability of sICH with mechanical thrombectomy was based on the MERCI study. ${ }^{14}$ The fraction of patients with sICH who were independent, dependent, or dead immediately after stroke treatment was based on the NINDS tPA stroke study results. ${ }^{12}$ In patients without SICH with recanalization, the fraction of individuals in each health state was based on the clinical outcomes of patients with successful recanalization in the MERCI study ${ }^{14}$ adjusted to reflect the absence of sICH. Similarly, in patients without sICH and without recanalization, the fraction of individuals in each health state was based on the clinical outcomes of patients without recanalization in the MERCI study, ${ }^{14}$ adjusted to reflect the absence of sICH (Fig. 1). The annual probability of dying was estimated for each health state based on the age-related mortality rate and the risk of a recurrent stroke.

We estimated quality-of-life factors for each health state based on a previously published costeffectiveness study (Table 1). The cost parameters used in our model were estimated from a variety of sources. United States hospitals collect reimbursements from Medicare for the care of stroke patients who undergo mechanical thrombectomy under MS-DRGs 023 and 024 . The total procedural and inpatient hospitalization costs for patients who underwent mechanical thrombectomy was estimated to be equal to the national average reimbursement for MS-DRGs 023 and 024, plus the average professional fees for CPT (Current Procedural Terminology) codes associated with this procedure. The cost of the hospitalization for patients who received standard medical therapy was estimated to be equal to the Medicare reimbursement for DRG 15 (acute ischemic stroke without thrombolysis or thrombectomy). ${ }^{5}$ Values for all other costs were adopted from the literature, ${ }^{4}$ and converted to 2008 US dollars using the medical care component of the consumer price index (Table 1).

\section{Sensitivity Analysis}

We assumed deterministic values for all model parameters. However, we performed univariate sensitivity analyses to evaluate the effects of varying each parameter on health and economic outcomes. For each parameter we considered a plausible range of values estimated from the 
literature. If no data from the literature were available, the deterministic value $\pm 25 \%$ was used as the range.

\section{Results}

For the base case scenario, standard medical therapy yielded 1.83 QALYs at a cost of $\$ 142,000$. Mechanical thrombectomy generated 2.37 QALYs at a cost of $\$ 148,600$. Therefore, mechanical thrombectomy resulted in a gain of 0.54 QALYs at an increased cost of $\$ 6600$, yielding an ICER of $\$ 12,120$ per QALY gained (Table 2).

Univariate sensitivity analyses indicated that the study results were robust. The ICER for mechanical thrombectomy remained $<\$ 50,000$ per QALY gained for all model inputs varied over a reasonable range, except for age at stroke treatment. Mechanical thrombectomy was considered borderline cost-effective (that is, ICER $<\$ 100,000$ per QALY gained) in patients older than 82 years of age. Although mechanical thrombectomy became less cost-effective as the age at initial treatment increased, the relative health benefits associated with this procedure remained constant: mechanical thrombectomy resulted in 30\% more QALYs than standard medical therapy across all ages tested. In addition to age, the ICER was most sensitive to the following model parameters: probability of recanalization with mechanical thrombectomy, hospitalization costs for patients receiving mechanical thrombectomy, and cost of long-term care for dependent patients (Fig. 2).

\section{Discussion}

These results suggest that mechanical thrombectomy performed for acute stroke within 8 hours of symptom onset is cost-effective from a US societal perspective. The incremental cost of mechanical thrombectomy over standard medical therapy is $\$ 12,000$ per QALY gained. This cost-effectiveness ratio is well below the generally accepted cost-effectiveness threshold of $\$ 50,000$ per QALY gained. ${ }^{8}$

Intravenous thrombolytic therapy with tPA for ischemic stroke has been shown to be a costsaving intervention by reducing health expenditures by $\$ 8000$ per QALY gained. ${ }^{6}$ Our findings are in line with the intravenous IPA cost-effectiveness data. The higher cost per QALY gained with mechanical thrombectomy is expected given 1) the higher cost of mechanical thrombectomy, and 2) the reduced chance of dramatic clinical improvement with mechanical thrombectomy, as this treatment is typically offered later, when recanalization is overall less effective. $^{9}$

The MERCI trial that led to FDA clearance of the MERCI clot retrieval system was an openlabel prospective cohort study without a control arm. ${ }^{14}$ The main limitation of our study is the lack of efficacy data from randomized controlled trials of mechanical thrombectomy in acute stroke. There is also no suitable historical control group comparable to the MERCI study population in terms of key baseline variables such as severity of stroke symptoms, time to treatment, and patient age. There is, however, a preponderance of evidence that mechanical thrombectomy is associated with higher recanalization rates than medical therapy, and that early recanalization is associated with improved clinical outcomes in patients with acute stroke. 10,14 It is also well-established that symptomatic intracerebral hemorrhage rates are higher in patients who undergo treatment with mechanical thrombectomy devices compared with those who receive standard medical therapy. ${ }^{13,14}$ Therefore, to model the cost-effectiveness of mechanical thrombectomy, a hypothetical control group was constructed that differed from the mechanical thrombectomy group by having a lower recanalization rate (18 vs $60 \%$ ) and a lower sICH rate (0.6 vs $7.8 \%)$. As illustrated in Fig. 1, the probabilities of each of the 3 health states (independent, dependent, or dead), conditional on recanalization and sICH status, were deemed 
identical for the control and mechanical thrombectomy arm. Although we have used the best method to determine cost-effectiveness with currently available data, our results require confirmation once efficacy data from randomized controlled trials become available. Two large North American randomized controlled acute stroke studies of mechanical thrombectomy, IMS (Interventional Management of Stroke) and MR Rescue (MR and Recanalization of Stroke Clots Using Embolectomy), are currently enrolling patients.

All model inputs were best estimates based on previously published data in peer-reviewed medical literature. It is possible, however, that these best estimates over- or underestimate the true value of any of the model parameters. Sensitivity analyses were performed to determine whether our main results would be significantly influenced by variations in model inputs. The results of the sensitivity analyses showed that our findings were robust against all model assumptions. For example, after varying the probability of recanalization over the published range, mechanical thrombectomy remained cost-effective (ICER $<\$ 50,000$ per QALY gained). A recanalization rate of $48 \%$ was used as the lower limit because this is the rate of recanalization with deployment of the mechanical device but without adjuvant therapy reported in the MERCI trial. At this recanalization rate the ICER was $\$ 23,385$ per QALY gained. For the base case a recanalization rate of $60 \%$ was used, based on the recanalization rate with mechanical thrombectomy and adjuvant thrombolytic therapy reported in the MERCI trial; at this rate the ICER was $\$ 12,120$ per QALY gained. Based on a systematic review, a recanalization rate of $84 \%$ was used as the upper limit in the sensitivity analysis, resulting in an ICER of $\$ 3946$ per QALY gained. ${ }^{10}$ The recanalization rate with the Penumbra device is $81 \%$ based on data in a recently presented abstract. ${ }^{11}$ As this is close to the upper range of the sensitivity analysis on the recanalization rate, the ICER for mechanical thrombectomy with the Penumbra device compared with standard medical therapy is $\sim \$ 4000$ per QALY gained. Age at the time of stroke treatment was the only variable for which the ICER exceeded $\$ 50,000$ in the sensitivity analysis. Mechanical thrombectomy became less cost-effective with advanced age and was borderline cost-effective (ICER \$50,000-100,000 per QALY gained) for patients older than 82 years of age.

\section{Conclusions}

Based on currently available data, mechanical thrombectomy performed within 8 hours of stroke onset increases survival by 0.54 QALYs and appears to be a cost-effective intervention (ICER of $\$ 12,000$ per QALY gained) compared with standard nonthrombolytic medical therapy. This cost-effectiveness estimate should be reassessed once data from randomized controlled trials of mechanical thrombectomy become available.

\section{Acknowledgements}

Disclosure The funding for this study was provided by national institutes of health (NIH) grants K23 NS051372 to Principal Investigator Maarten G. Lansberg.

\section{Abbreviations used in this paper}

FDA, Food and Drug Administration; ICER, incremental cost-effectiveness ratio; MERCI, Mechanical Embolus Removal in Cerebral Ischemia; mRS, modified Rankin Scale; MS-DRG, Medicare Severity Diagnostic-Related Group; NINDS, National Institute of Neurological Disor ders and Stroke; PROACT, Pro-Urokinase for Acute Ce rebral Thromboembolism; QALY, quality-adjusted life year; sICH, symptomatic intracerebral hemorrhage; tPA, tissue plasminogen activator. 


\section{References}

1. The National Institute of Neurological Disorders and Stroke rt-PA Stroke Study Group. Tissue plasminogen activator for acute ischemic stroke. N Engl J Med 1995;333:1581-1587. [PubMed: 7477192]

2. Adams HP Jr, del Zoppo G, Alberts MJ, Bhatt DL, Brass L, Furlan A, et al. Guidelines for the early management of adults with ischemic stroke: a guideline from the American Heart Association/ American Stroke Association Stroke Council, Clinical Cardiology Council, Cardiovascular Radiology and Intervention Council, and the Atherosclerotic Peripheral Vascular Disease and Quality of Care Outcomes in Research Interdisciplinary Working Groups: The American Academy of Neurology affirms the value of this guideline as an educational tool for neurologists. Circulation 2007;115:e478e534. [PubMed: 17515473]

3. Albers GW, Amarenco P, Easton JD, Sacco RL, Teal P. American College of Chest Physicians: Antithrombotic and thrombotic therapy for ischemic stroke: American College of Chest Physicians evidence-based clinical practice guidelines (8th edition). Chest 2008;133:630-669.

4. Chambers MG, Koch P, Hutton J. Development of a decision-analytic model of stroke care in the United States and Europe. Value Health 2002;5:82-97. [PubMed: 11918824]

5. Demaerschalk BM, Durocher DL. How diagnosis-related group 559 will change the US Medicare cost reimbursement ratio for stroke centers. Stroke 2007;38:1309-1312. [PubMed: 17332446]

6. Fagan SC, Morgenstern LB, Petitta A, Ward RE, Tilley BC, Marler JR, et al. NINDS rt-PA Stroke Study Group. Cost-effectiveness of tissue plasminogen activator for acute ischemic stroke. Neurology 1998;50:883-890. [PubMed: 9566367]

7. Furlan A, Higashida R, Wechsler L, Gent M, Rowley H, Kase C, et al. Intra-arterial prourokinase for acute ischemic stroke. The PROACT II study: a randomized controlled trial. Prolyse in Acute Cerebral Thromboembolism. JAMA 1999;282:2003-2011. [PubMed: 10591382]

8. Gold, MR.; Siegel, JE.; Russell, L.; Weinstien, M. Cost-Effectiveness in Health and Medicine. Oxford University Press; New York: 1996.

9. Hacke W, Donnan G, Fieschi C, Kaste M, von Kummer R, Broderick JP, et al. Association of outcome with early stroke treatment: pooled analysis of ATLANTIS, ECASS, and NINDS rt-PA stroke trials. Lancet 2004;363:768-774. [PubMed: 15016487]

10. Rha JH, Saver JL. The impact of recanalization on ischemic stroke outcome: a meta-analysis. Stroke 2007;38:967-973. [PubMed: 17272772]

11. McDougall, C.; Clark, W.; Mayer, T.; Doertler, A.; Grunwald, I.; Berkefeld, J., et al. The Penumbra Stroke Trial: safety and effectiveness of a new generation fo mechanical devices for clot removal in acute ischemic stroke, abstract presented at the International Stroke Meeting,; New Orleans, LA. February 20-22; 2008.

12. Saver JL. Hemorrhage after thrombolytic therapy for stroke: the clinically relevant number needed to harm. Stroke 2007;38:2279-2283. [PubMed: 17641238]

13. Smith WS. Safety of mechanical thrombectomy and intravenous tissue plasminogen activator in acute ischemic stroke. Results of the multi Mechanical Embolus Removal in Cerebral Ischemia (MERCI) trial, part I. AJNR, Am, J, Neuroradiol 2006;27:1177-1182. [PubMed: 16775259]

14. Smith WS, Sung G, Starkman S, Saver JL, Kidwell CS, Gobin YP, et al. Safety and efficacy of mechanical embolectomy in acute ischemic stroke: results of the MERCI trial. Stroke 2005;36:14321438. [PubMed: 15961709] 


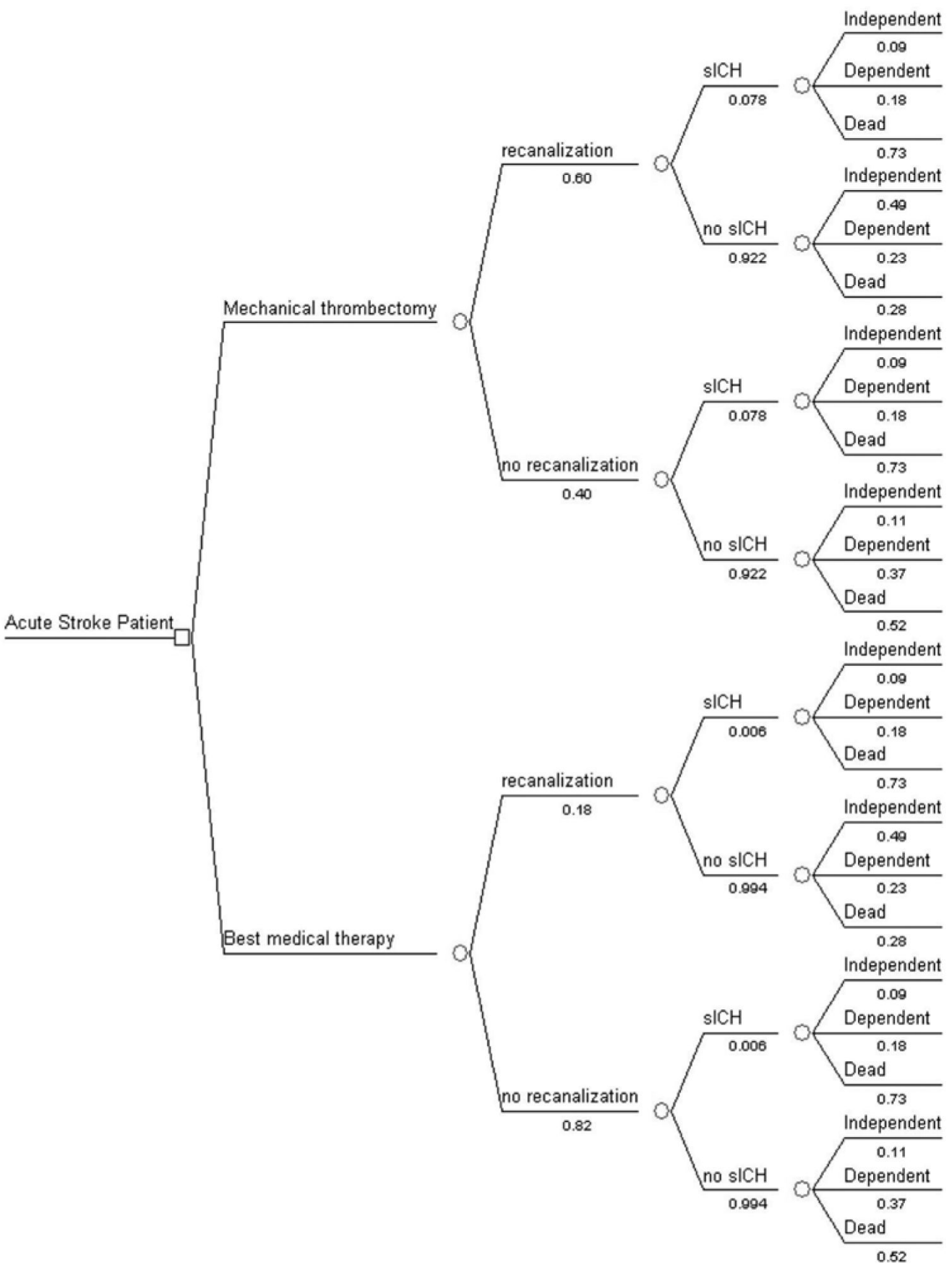

Fig. 1.

Flow-chart diagram of the decision tree that was used to model cost-effectiveness of mechanical thrombectomy versus standard medical therapy in patients with acute ischemic stroke. 


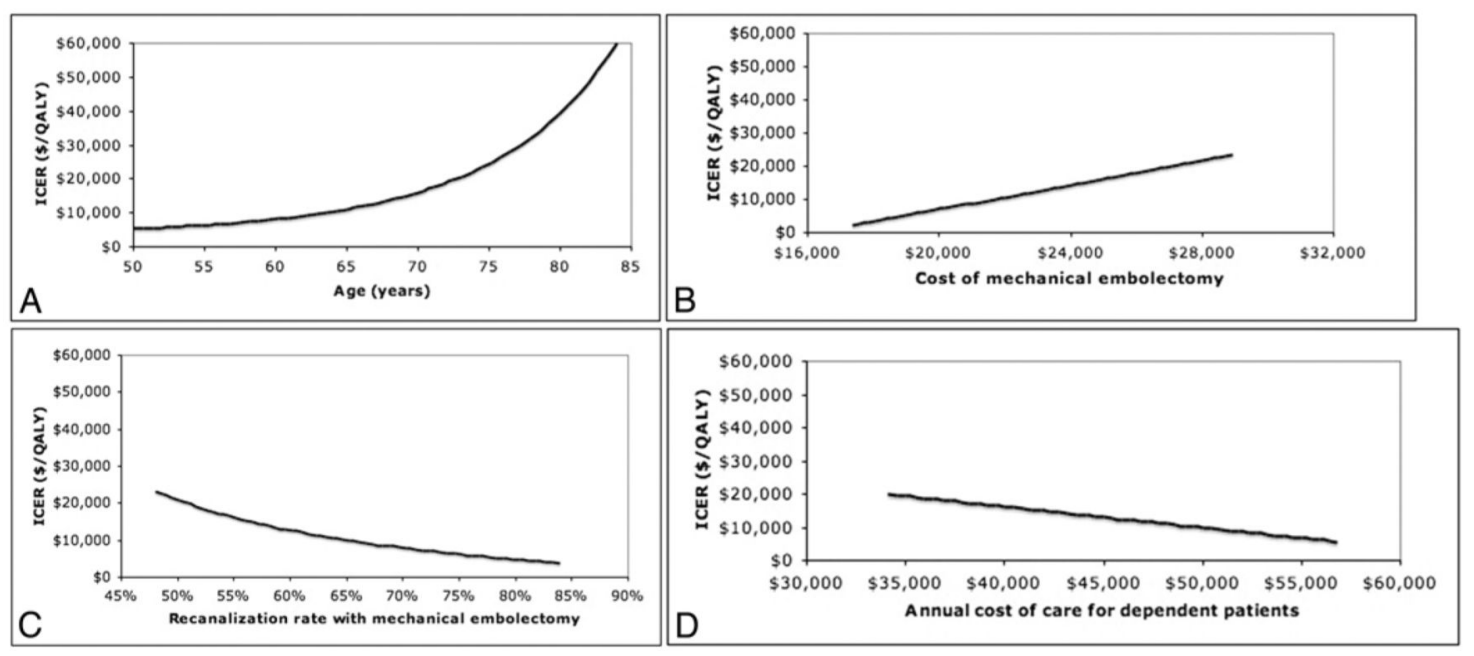

Fig. 2.

Graphs of univariate sensitivity analyses demonstrating the impact of 4 model inputs on the ICER: age at stroke treatment (A), cost of hospitalization for patients who undergo mechanical thrombectomy (B), probability of recanalization with mechanical thrombectomy $(\mathrm{C})$, and cost of long-term care for dependent patients (D). 
TABLE 1

Probabilities, utilities, and costs used in the model, and the range of values tested in the univariate sensitivity analyses*

\begin{tabular}{|c|c|c|c|}
\hline Model Input & Standard Therapy (range) & Mechanical Thrombectomy (range) & Reference No. \\
\hline \multicolumn{4}{|l|}{ probability of } \\
\hline recanalization & $0.18(0.18-0.24)$ & $0.60(0.48-0.84)$ & $5,8,12,13$ \\
\hline sICH & $0.006(0.006-0.020)$ & $0.078(0.078-0.110)$ & $1,5,10,13$ \\
\hline recurrent stroke & $0.052(0.039-0.065)$ & $0.052(0.039-0.065)$ & 9 \\
\hline \multicolumn{4}{|l|}{ utility (QALY) } \\
\hline $\begin{array}{l}\text { independent (mRS score } \leq \\
\text { 2) }\end{array}$ & $0.74(0.55-0.92)$ & $0.74(0.55-0.92)$ & 9 \\
\hline dependent (mRS score 3-5) & $0.40(0.30-0.50)$ & $0.40(0.30-0.50)$ & 9 \\
\hline dead & 0 & 0 & 9 \\
\hline \multicolumn{4}{|l|}{$\operatorname{cost}(\$)$} \\
\hline $\begin{array}{l}\text { total cost of hospitalization } \\
\text { for acute stroke }\end{array}$ & $6749(5061-8436)$ & $24,154(18,365-29,942)$ & 3 \\
\hline $\begin{array}{l}\text { annual care for } \\
\text { independent patients }\end{array}$ & $5764(4323-7205)$ & $5764(4323-7205)$ & 2 \\
\hline $\begin{array}{l}\text { annual care for dependent } \\
\text { patients }\end{array}$ & $45,469(34,101-56,836)$ & $45,469(34,101-56,836)$ & 2 \\
\hline $\begin{array}{l}\text { hospitalization for } \\
\text { recurrent stroke }\end{array}$ & $24,939(18,704-31,173)$ & $24,939(18,704-31,173)$ & 2 \\
\hline
\end{tabular}




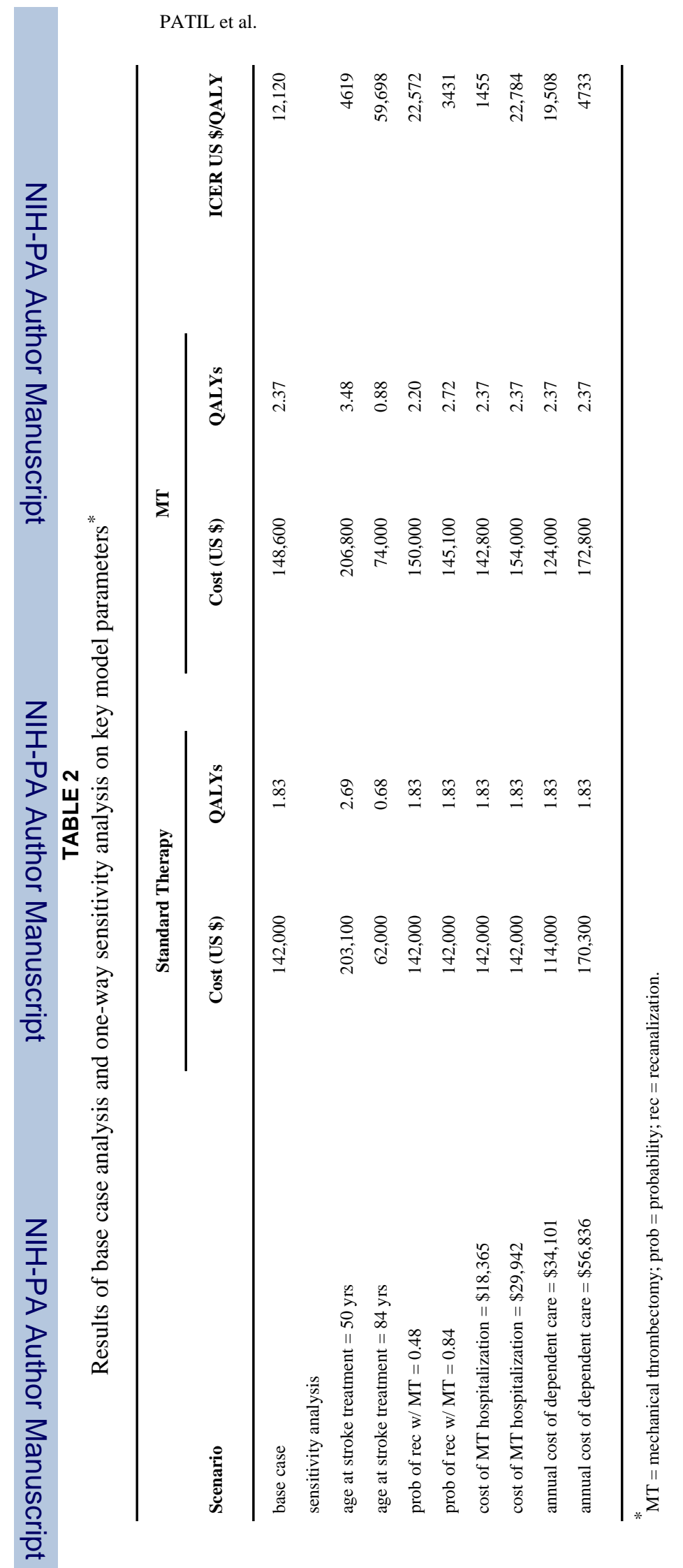

J Neurosurg. Author manuscript; available in PMC 2010 March 1. 